\title{
Síndrome de Hellp - complicação da pré-eclâmpsia: Um relato de experiência
}

\author{
Hellp syndrome- pre-eclampsy complication: An experience report \\ Síndrome de Hellp - complicación pre-eclampsia: Un informe de experiencia
}

\author{
Pâmela Souza Bastos \\ ORCID: https://orcid.org/0000-0002-7886-8331 \\ Faculdade Irecê, Brasil \\ E-mail: pamelajp.1024@ hotmail.com \\ Cláudio José Dourado de Almeida \\ ORCID: https://orcid.org/0000-0002-5757-1010 \\ Faculdade Irecê, Brasil \\ E-mail: claudiojda@gmail.com \\ Diógenes Vaz de Oliveira \\ ORCID: https://orcid.org/0000-0002-8145-2361 \\ Faculdade Irecê, Brasil \\ E-mail: diogenes.vaz@faifaculdade.com.br \\ Cintia Ferreira Amorim \\ ORCID: https://orcid.org/0000-0002-4650-8278 \\ Faculdade Irecê, Brasil \\ E-mail: cintiaferreiraamorim31@gmail.com \\ Hebe Raquel Cardoso Souza Dourado \\ ORCID: https://orcid.org/0000-0002-4518-1787 \\ Faculdade Irecê, Brasil \\ E-mail: raquelcdourado2@gmail.com
}

\begin{abstract}
Resumo
Objetivo: Descrever sobre a assistência realizada a mulher com Síndrome de Hellp. Metodologia: Trata-se de um estudo de caráter descritivo e natureza qualitativa na modalidade relato de experiência. Contudo, a pesquisa foi realizada a partir da vivência de uma discente do curso de Bacharelado em enfermagem, durante o estágio curricular, realizado em uma unidade hospitalar situada no interior da Bahia. Resultados: As gestantes com pré-eclâmpsia e hipertensão gestacional eram classificadas em vermelho, para aquelas apresentavam convulsão ou trabalho de parto em período expulsivo, laranja para aquelas gestantes com níveis pressóricos $\geq 140 \times 100 \mathrm{mmHg}$ apresentando_cefaleia epigastralgia e escotoma, e níveis pressóricos $\geq 160 \times 100 \mathrm{mmHg}$, e essa classificação é realizada pelo enfermeiro. Durante o atendimento a gestante grave, foi possível identificar gestantes com pré-eclâmpsia que não eram acompanhadas no prénatal de alto risco, e mesmo sendo atendida no pré-natal de baixo risco não faziam uso de anti-hipertensivo para controle dos níveis pressóricos. Conclusão: É evidente que ainda existam lapsos relacionados ao tratamento da gestante com síndrome de Hellp, já que essa complicação não tem uma fisiopatologia concreta, e que é considerada por pesquisadores da área da obstetrícia como uma complicação da pré-eclâmpsia grave.
\end{abstract}

Palavras-chave: Complicações na gravidez; Pré-eclâmpsia; Gravidez de alto risco; Complicações hematológicas na gravidez.

\begin{abstract}
Objective: To describe the assistance provided to women with Hellp Syndrome. Methodology: This is a study of a descriptive character and qualitative nature in the experience report modality. However, the research was carried out from the experience of a student of the Bachelor of Nursing course, during the internship, held in a hospital located in the interior of Bahia. Results: Pregnant women with pre-eclampsia and gestational hypertension were classified in red, for those with seizures or labor in expulsive period, orange for those with blood pressure $>140 \times 100 \mathrm{mmHg}$, with epigastric pain and scotoma headache, and blood pressure $>160 \times 100 \mathrm{mmHg}$, and this classification is performed by the nurse. During the care of a serious pregnant woman, it was possible to identify pregnant women with pre-eclampsia who were not followed up in high-risk prenatal care, and even being attended to in low-risk prenatal care, they did not use antihypertensive drugs to control their blood pressure levels. Conclusion: It is evident that there are still lapses related to the treatment of pregnant women with Hellp syndrome, since this complication does not have a concrete pathophysiology, and which is considered by researchers in the area of obstetrics as a complication of severe preeclampsia.
\end{abstract}

Keywords: Pregnancy complications; Pre eclampsia; High-risk pregnancy; Haematological complications in pregnancy. 


\section{Resumen}

Objetivo: Describir la asistencia brindada a mujeres con Síndrome de Hellp. Metodología: Se trata de un estudio de carácter descriptivo y cualitativo en la modalidad de relato de experiencia. Sin embargo, la investigación se realizó a partir de la experiencia de una estudiante de la carrera de Licenciatura en Enfermería, durante la pasantía curricular, realizada en un hospital ubicado en el interior de Bahía. Resultados: Las mujeres embarazadas con preeclampsia e hipertensión gestacional se clasificaron em rojo, para aquellas con convulsiones o trabajo de parto em período expulsivo, naranja para aquellas con presión arterial $>140 \times 100 \mathrm{mmHg}$, con dolor epigástrico y cefalea escotoma, y presión arterial >160x100 mmHg, y esta clasificación la realiza la enfermería. Durante el cuidado de una gestante grave se logró identificar a gestantes con preeclampsia que no fueron seguidas en atención prenatal de alto riesgo, e incluso siendo atendidas en atención prenatal de bajo riesgo, no utilizaron medicamentos antihipertensivos para controlar sus niveles de presión arterial. Conclusión: Es evidente que aún existen lapsos relacionados con el tratamiento de gestantes con síndrome de Hellp, ya que esta complicación no tiene una fisiopatología concreta, y es considerada por investigadores del área de obstetricia como una complicación de la preeclampsia grave.

Palabras clave: Complicaciones del embarazo; Preeclampsia; Embarazo de alto riesgo; Complicaciones hematológicas en el embarazo.
\end{abstract}

\title{
1. Introdução
}

A Hipertensão arterial sistêmica (HAS) durante a gestação é responsável pelo maior índice de mortalidade maternoinfantil, além de ser considerada como um problema de saúde pública, visto que, a Síndrome de Hellp é caracterizada como uma complicação da pré-eclâmpsia, que pode ocorrer entre 24-48 horas após o parto, e apresentam-se em três critérios tais como: hemólise, aumento das enzimas hepáticas, e plaquetopenia (Gonçalves, Pereira, Oliveira, \& Gonzaga, 2018; Ribeiro, Melo, Silva, Guimarães, \& Santos, 2017).

Vale ressaltar, que essa patologia foi relatada pela primeira vez no ano de 1982 pelo pesquisador Louis Weinsten, o qual ele observou 29 mulheres com diagnóstico de pré-eclampsia, essas gestantes apresentaram trombocitopenia, hemólise, e alterações hepáticas (Gonçalves et al., 2018).

De acordo com a Organização Mundial de Saúde a Síndrome de Hellp é responsável por um quarto dos óbitos maternos da América Latina, já que a gestação é considerada como um processo fisiológico e prazeroso na vida das mulheres, porém em alguns momentos essa fase da mulher toma um rumo desfavorável, com isso a gestação se torna de alto risco, e necessita de um acompanhamento de pré-natal rigoroso para identificação precoce dessas alterações (Krebs, Silva, \& Bellotto, 2021).

Nessa perspectiva, com base nesse estudo o pesquisador propôs que as mulheres que apresentassem essas alterações fossem distinguidas daquelas com diagnóstico de pré-eclâmpsia grave, sendo denominado de Síndrome de Hellp, em relação ao termo HELLP, este é um acrônimo das abreviações dos termos em inglês que querem dizer: H - hemolysis (hemólise), EL elevated liver enzymes (aumento das enzimas hepáticas) LP - low platelets (plaquetopenia) (Ribeiro et al., 2017).

Dado isso, sua fisiopatologia ainda não foi totalmente desmistificada, porém acredita-se que ocorre redução na perfusão placentária, falha na invasão do trofoblastos, e assim altera a função do endotélio, ocasionando um processo inflamatório, e queda dos níveis de prostaglandinas e aumento da ação do tromboxano resultando em avanço da atividade vascular, o qual pode ocasionar complicações maternas, como insuficiência cardíaca, pulmonar, renal, fetal, crescimento uterino restrito e Síndrome da Angústia Respiratória (Pereira et al., 2016).

Atrelado a isso, a Síndrome de Hellp pode ser classificada em completa classe I, II, o qual ocorre alterações plaquetárias $<100.000$ plaquetas por $\mathrm{ml}$, DHL $\leq 600 \mathrm{UI} / \mathrm{L}$ ou billirrubina $\geq 1,2 \mathrm{mg} / \mathrm{dl}$ ou esquizócitos, TGO $\geq 70 \mathrm{UI} / \mathrm{L}$, podendo ser classificada como incompleta quando a paciente apresenta uma ou duas das alterações citadas anteriormente, parcial classe III nos casos de pré-eclâmpsia grave + ausência de hemólise, alterações das enzimas hepáticas, plaquetas entre $100.000 \mathrm{~mm}^{3}$ e $150.000 \mathrm{~mm}$ (Brasil., 2010; Oliveira et al., 2012; Santos et al., 2020). 
Denota-se, que até o presente momento a patologia não dispõe de tratamento específico, o mesmo é conduzido conforme os sintomas que a paciente apresenta, o qual se tem pouco conhecimento sobre a fisiopatologia da doença, porém o parto e a remoção dos vilos coriônicos são adotados como tratamento definitivo da síndrome de Hellp (Ribeiro et al., 2017).

Precipuamente, vale destacar a importância da assistência pré-natal à gestante de risco, que deve ser discernido quando comparadas às gestantes sem riscos. Desse modo é necessária a identificação precoce dessas alterações, acompanhamento dos níveis pressóricos e sintomas que a gestante pode relatar durante as consultas de pré-natal. A gestante de alto risco deve ter um atendimento diferenciado desde o número e qualidade das consultas, pois esse atendimento objetiva-se minimizar riscos e desfechos desfavoráveis (Brasil, 2012; Pio, Peraçoli, \& Bettini, 2019).

Neste contexto, surgiu o interesse em realizar essa pesquisa a partir da experiência adquirida como acadêmica de enfermagem durante a prática do estágio curricular em Unidade Hospitalar, mediante ao atendimento de enfermagem prestado a gestante com Síndrome de Hellp, e assim contribuir para a ciência informações relevantes relacionadas ao tema, já que o mesmo possui algumas lacunas a serem desvendadas. Desta forma objetiva-se descrever sobre a assistência realizada a mulher com Síndrome de Hellp.

\section{Metodologia}

Trata-se de um estudo de caráter descritivo e natureza qualitativa na modalidade relato de experiência, o qual é considerado um estudo metodológico que permite descrever experiências vivenciadas pelo autor (Gil, 2010). Contudo, a pesquisa foi realizada a partir da vivência de uma discente do curso de Bacharelado em enfermagem, durante o estágio curricular, realizado em uma unidade hospitalar situada no interior da Bahia.

Graduando do décimo semestre do curso de enfermagem se configura como autora desse relato, detalhando sua trajetória vivenciada no estágio Supervisionado II, com gestantes diagnosticadas com pré-eclâmpsia durante o pré-natal, os quais evoluíram para complicação, com Síndrome de Hellp. A experiência ocorreu no mês de março do ano de 2021 de segunda a sexta-feira, sendo prestada assistência às gestantes e puérperas da clínica obstétrica, sob a orientação de um supervisor.

A população estimada onde ocorreu a prática curricular é composta de 73.524 habitantes conforme o Instituto Brasileiro de Geografia e Estatísticas (IBGE), visto que, os leitos da unidade atendem a central estadual de regulação, sendo transferido paciente de todo o estado da Bahia conforme a regulação.

\section{Resultados e Discussão}

\subsection{Classificação de risco a gestante com pré-eclâmpsia/síndrome de Hellp}

A classificação de risco das gestantes com síndrome hipertensiva gestacional (pré-eclâmpsia/eclâmpsia, síndrome de Hellp) é realizada na triagem obstétrica, com base no protocolo de Manchester (Fernades, 2010), além de ser utilizado Procedimento Operacional Padrão (POP) da instituição. Durante os atendimentos na triagem obstétrica foi possível realizar sinais vitais, punção de acesso venoso periférico e observar condutas obstétricas.

Vale ressaltar, que o protocolo de Manchester é utilizado da seguinte maneira: vermelho para pacientes grave ou em trabalho de parto ativo, laranja para pacientes com condições que pode agravar sem o atendimento, amarelo para aquelas que possuem condições clínicas para aguardar o atendimento, verde baixo risco de agravo, e azul sem risco (Fernades, 2010).

Dado isso, o POP é composto por fluxograma para acolhimento da gestante na classificação de risco composto por grupos: o primeiro grupo, prioridade máxima (emergência) atendimento imediato, grupo dois, prioridade I muito urgente atendimento em até 10 minutos, terceiro grupo, prioridade II urgente atendimento em até 30 minutos, quarto grupo, prioridade III pouco urgente atendimento em até 120 minutos, quinto grupo, prioridade IV não urgente atendimento em até quatro horas. 
As gestantes com pré-eclâmpsia e hipertensão gestacional eram classificadas em vermelho, para aquelas que apresentavam convulsão ou trabalho de parto em período expulsivo, laranja para aquelas gestantes com níveis pressóricos

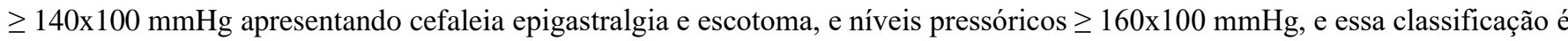
realizada pelo enfermeiro do setor.

Paralelo a isso, foi possível perceber o quanto a assistência de enfermagem é importante nesse processo de classificação de risco das gestantes de acordo os sinais e sintomas que a paciente apresenta, além da coleta de dados detalhada. Durante o atendimento, era solicitada a caderneta da gestante, porém a maioria não possuía dados precisos e estavam incompletos.

De acordo Milanes, Suárez, Castro, \& Hurtado (2019), destacam que os sinais e sintomas da síndrome de Hellp são variáveis. Vale elencar, que quando a gestante apresenta algia epigástrica no quadrante superior direito do abdômen a partir da segunda metade da gravidez pode evoluir para síndrome de hellp, porém aquelas gestantes com pré-eclâmpsia e dor epigástrica intensa em região de abdômen direto a mesma pode está apresentando sangramento com hematoma subcapsular ou ruptura do fígado.

Como a síndrome Hellp é considerada uma doença inespecífica ainda na literatura outros sintomas não clássicos podem estar associados aos diagnósticos como: edema agudo de pulmão, ascite, derrame pleural e diabetes gestacional Mellitus e servem de alerta durante a abordagem inicial para garantia de um diagnóstico mais rápido (Lopes et. al, 2013).

Foi possível identificar gestantes com pré-eclâmpsia que não eram acompanhadas no pré-natal de alto risco, e mesmo sendo atendidas no pré-natal de baixo risco não faziam uso de anti-hipertensivo para controle dos níveis pressóricos. Ainda convém destacar que durante o acolhimento dessas pacientes os níveis pressóricos se mantinham entre 160X90 mmhg 180X100 mmhg.

De igual modo, Moura et al. (2020), considera importante a prevenção de pré-eclâmpsia no pré-natal, com base em intervenções farmacológicas em tempo hábil, orientações quanto ao estilo de vida saudável, no entanto as estratégias para diminuir o risco de hipertensão na gravidez são consideradas como uma prioridade global.

No pré-natal, a gestante desde a primeira consulta deve ser investigada sobre fatores de risco para hipertensão e ser orientada sobre medidas de como conter o controle pressórico como descrito na diretriz Brasileira de hipertensão, medidas preventivas têm impacto imprescindível no aparecimento de complicações (Oigman, W., \& Nadruz, W., 2021).

Embora, essa Síndrome sendo considerada como uma complicação da pré-eclâmpsia, até seu reconhecimento como uma patologia relacionada a uma complicação de outra doença, era confundida e diagnosticada como quadros de hepatite, colecistite, culminado em desfechos desfavoráveis para a gestante e feto, devido diagnósticos tardios (Oliveira et al., 2012).

Ainda convém lembrar que a Organização Mundial de Saúde (OMS) estima que a incidência de pré-eclâmpsia ocorre sete vezes mais em países de classe média do que em países de classe alta, tendo em vista que, o risco de uma gestante morrer com pré-eclâmpsia/eclâmpsia é 300 vezes mais do que aquelas que vivem em países de alta renda (Malmstrom, Haberg, \& Morken, 2020).

Ficou evidente que grandes quantidades de mulheres atendidas faziam parte da população de classe média e classe baixa, notório baixo grau de informações relacionadas a gravidez e riscos que tais patologias poderiam ocasionar durante parto e puerpério, algumas delas apresentavam na caderneta de gestante consultas de pré-natal de rotina conforme idade gestacional.

\subsection{Atendimento a gestante com pré/eclampsia e síndrome de Hellp na clínica obstétrica}

Por se tratar de gestantes grave, durante o estágio curricular foi possível prestar atendimento com base na Sistematização da Assistência de Enfermagem (SAE), embasando um cuidado acolhedor e preciso, e identificado a importância do enfermeiro no processo de gerenciamento do setor, visto que é composto por uma equipe multidisciplinar (médico obstetra, nutricionista, assistente social, serviços gerais entre outros). 
Com base nos sinais e sintomas que cada paciente apresentava o plano de cuidados era elaborado com base no Diagnóstico de enfermagem da NANDA-I (2018), como risco de infecção, risco de infecção em sítio cirúrgico, dor no trabalho de parto, dor aguda, baixa autoestima, volume de líquido excessivo, náuseas, privação do sono, eliminações vesicais prejudicadas, constipação, risco de sangramento, risco de função hepática prejudicada, risco de glicemia instável, medo.

Em vista disso, compreende a relevância da utilização da sistematização da assistência de enfermagem, com intuito de implementar o processo de enfermagem no acolhimento e assistência a essa gestante de alto risco, além de possibilitar o levantamento dos diagnósticos e intervenção de enfermagem, que visam não apenas à qualidade da assistência prestada, mas também, ampliação e a visibilidade do reconhecimento profissional (Oliveira et al., 2018).

Em contrapartida, vale destacar que na Síndrome de hellp são adotadas três condutas tais como: a primeira é a interrupção imediata da gravidez, indicada quando a paciente apresenta eclâmpsia, sofrimento fetal agudo, coagulopatia, hellp classe I, complicações da pré-eclâmpsia (hipertensão refratária, insuficiência renal, iminência de eclâmpsia e em gestação acima de 34 semanas), a segunda conduta baseia-se em uso de corticóides administrados a mãe com o objetivo de acelerar a maturidade pulmonar fetal, e está indicada entre 24 e 34 semanas de gestação, e a terceira conduta considerada como conservadora onde é feita monitorização materno-fetal em centros terciários (Oliveira et al., 2012).

Assunto esse, de grande controvérsia na obstetrícia, tendo em vista que, esse procedimento deve ser realizado conforme a idade gestacional, e avaliações maternas e fetais. A gestante com idade gestacional maior ou igual há 37 semanas, necessita de estabilização para que possa ser realizada a interrupção da gestação de forma segura, de acordo com a conduta obstétrica. Para os casos graves é necessário internamento com monitorização rigorosa dos níveis pressóricos, acompanhamento clínicolaboratorial, avaliação do bem-estar fetal (Pozza, Delaney, Burmam, \& Paula, 2018).

As pacientes conduzidas para a clínica obstétrica no hospital do interior da Bahia, eram pacientes que geralmente vinham do centro cirúrgico já estabilizado do quadro clínico, pois a partir do atendimento na classificação de risco e avaliação do obstetra eram encaminhadas para interrupção da gestação, porém alguns casos eram encaminhados para a clínica para monitorização e acompanhamento.

O acolhimento humanizado pela equipe de enfermagem a gestante grave na clínica obstétrica, caracteriza-se pela avaliação do enfermeiro a cada 1/1 hora de acordo com a dinâmica do parto, é observado contrações, batimentos cardiofetais (BCF), sinais vitais, sangramentos importantes, e queixas que a gestante pode relatar, além de avaliar exames laboratoriais caso ocorra alterações é solicitada avaliação do médico obstetra de plantão.

Associado a isso, com base na pesquisa realizada por Ribeiro et al. (2017), destacam que são adotadas as seguintes medidas, na assistência à gestante com pré-eclâmpsia/eclâmpsia e síndrome de hellp, tais como: acondicionar a gestante em ambiente tranquilo, com pouca luminosidade, cabeceira elevada para conservar vias aéreas pérvias, cabeça lateralizada, oxigenioterapia, cânula de Guedel, cateterismo vesical de demora, acesso venoso periférico calibroso, terapia anticonvulsivante (sulfato de magnésio), terapia antihipertensiva (nifedipino, hidralazina etc.), avaliação da vitalidade fetal e avaliação laboratorial.

O enfermeiro se destaca como o protagonista assistencial durante a gestação, visto que, deve sempre estar atento aos sinais e sintomas que a gestante transmitir, desde a realização do pré-natal de qualidade e a colaboração da gestante com seus deveres, obrigações e limitações, além do encaminhamento e assistência prestada pela equipe multidisciplinar (médico obstetra, nutricionista entre outros profissionais) no momento certo (Oliveira et al., 2018).

Desse modo, enfatizo quão grande foi a importância do estágio curricular na assistência prestada à gestante com préeclâmpsia e síndrome de Hellp, no processo de graduação, visto que o campo de estágio nos proporcionou experiência marcante quando se trata de gestante grave, além de atendimento com gestantes de baixo grau. 


\section{Conclusão}

É evidente que ainda existam lapsos relacionados ao tratamento da gestante com síndrome de hellp, já que essa complicação não tem uma fisiopatologia concreta, e que é considerada por pesquisadores da área da obstetrícia como uma complicação da pré-eclâmpsia grave, desse modo o diagnóstico e tratamento desta adversidade se torna desafiador para os profissionais, tendo em vista que o atendimento a essas pacientes deve ser realizado com muita cautela.

Dado o exposto com o que foi mencionado anteriormente, o intuito do estudo é instigar o leitor a buscar embasamento científico relacionado à temática, com o objetivo de promover assistência acolhedora e qualificada a essas gestantes, visto que é uma doença que promove angústias e medos, pois a gestação é responsável por promover uma gama de sensações e emoções.

Em virtude aos fatos mencionados, é de grande relevância a promoção de pesquisa científica e estudo de campo relacionado ao diagnóstico e tratamento da síndrome de hellp, pois se trate de um tema relevante e de grande destaque para obstetrícia. Levando-se em consideração esses aspectos, esse trabalho instiga o leitor a necessidade de ir além do protocolo e do senso comum, deixando de ser tecnicista e buscando sempre uma atenção integral e humanizada às gestantes.

Conclui-se, que o estágio curricular contribuiu de forma relevante na construção de um futuro profissional com olhar holístico, visto que a assistência prestada a essas pacientes foi embasada através de conhecimento científico adquirido ao longo da graduação.

\section{Referências}

Brasil. Ministério da Saúde. (2010). Gestação de Alto Risco Manual Técnico (5a ed.).

Brasil. Ministério da Saúde. (2012). Secretaria de Atenção à Saúde. Departamento de Ações Programáticas Estratégicas. Gestação de Alto Risco: manual técnico/ Ministério da Saúde, Secretaria de Atenção à Saúde, Departamento de Ações Programáticas Estratégicas. 5 ed. Brasília: Editora do Ministério da Saúde, 302.

Fernades, S.A. (2010). Protocolos no serviço de urgência. Revista Nursing. p. 31-34.

Garcez, R. M., Barros, L. B. L., Napoleão, A. A., Cruz, D. L. A. M., Avena, M. J., \& Brasil, V. V. Diagnósticos de enfermagem da NANDA-I: definições e classificação 2018-2020. Artmed.

Gil, A. C. (2010). Como Elaborar Projetos de Pesquisa. Atlas.

Gonçalves, A. P. O. G., Pereira, P. S., Oliveira, V. C., \& Gonzaga, M. F. N. (2018). Síndrome de hellp: entendendo e intervindo. Revista Saúde em Foco, (10), 274.

H., Oigman, W., \& Nadruz, W. (2021). Diretrizes Brasileiras de Hipertensão Arterial - 2020. Arq.

Instituto Brasileiro de Geografia e Estatística (IBGE). Panorama da cidade de Irecê na Bahia.

Krebs, V. A., Silva, M. R., \& Bellotto, P. C. B. (2021). Síndrome de Hellp e Mortalidade Materna: Uma revisão integrativa. Brazilian Journal of Health Review. 4 (2). 6297-6311.

Lopes, G. T, Oliveira, M. C. R., Silva, K. M., Silva, I. F., Ribeiro, A. P. L. P. (2013). Hipertensão gestacional e a síndrome Hellp: ênfase nos cuidados de enfermagem. Revista Augustus. 18 (36). 77-89.|

Malmstrom, O., Haberg, S. E., \& Morken, N. H. (2020). Probabilidade e resultados da segunda gravidez após a síndrome HELLP na primeira: um estudo de registro de base populacional. Acta Obstet Gynecol Scand. 1 (8). https://doi.org/10.1590/S0100-72031998000300008

Milanes, G. T., Suárez, A. L. M., Castro, I. M., \& Hurtado, J. M. T. (2019). Síndrome de Hellp. Apresentação de um caso e revisão da entidade. Revista Médica. Granma. 23 (4).

Moura, N. S., Gomes, M. L. S., Rodrigues, I. R., Rolnik, D. L., Costa, F. S., \& Oriá, M. O. B. (2020). Procedimentos clínicos para a prevenção de pré-eclâmpsia em gestantes: Uma revisão sistemática. Revista Brasileira de Ginecologia e Obstetrícia. 42 (10). https://doi.org/10.1055/s-0040-1714135

Oliveira, L. A. M., Galvão, M. P. S. P., Soares, Y. K. C., Martins, C. R., Vasconcelos, B. P., Galvão, T. C. C. P., Neta, M. J. S., Leite, M. F. F. S., Noleto, L. C., \& Paula, M. M. (2018). Cuidados de Enfermagem a gestante com síndrome hipertensiva: revisão integrativa. Revista Brasileira de Cirurgia e Pesquisa Clínica - BJSCR. 23 (2), 159-164.

Oliveira, R. S., Matos, I. C., Silva, T. B. P., Azevedo, N. M., Andrade, M., \& Espirito Santo, F. H. (2012). Síndrome Hellp: estudo de revisão para o cuidado de enfermagem. Revista enfermeria global. 28.

Pereira, B., Linhares, G. M. M., Severo, I. M. M., Serra, J. L. G., Dutra, M., Quintão, R. A., \& Carvalho, J. (2016). Síndrome hellp: uma revisão de literatura. Revista da Faculdade de Medicina de Teresópolis 3 (2). 
Research, Society and Development, v. 10, n. 8, e20610817106, 2021

(CC BY 4.0) | ISSN 2525-3409 | DOI: http://dx.doi.org/10.33448/rsd-v10i8.17106

Pio, D. A. M., Peraçoli, J. C., \& Bettini, R. V. (2019). Vivências psíquicas de mulheres com pré-eclâmpsia: um estudo qualitativo. Revista Psicologia e Saúde, 11(2), 115-127. https://dx.doi.org/10.20435/pssa.v11i2.648

Pozza, L. V., Delaney, L. A., Burmann, L., \& Paula, L. G. (2018). Crise hipertensiva gestacional.

Ribeiro, J. F., Melo, S. S. S., Silva, C. C., Guimarães, S. V. C., \& Santos, T. M. M. G. (2017). Síndrome hellp: caracterização obstétrica e modalidade de tratamento. Revista de enfermagem UFPE on line, 11 (3).

Santos, M. R. P. P. N., Oliveira, A. H. A., \& Souza, P. G. V. D. (2020). A importância dos exames laboratoriais para o diagnóstico diferencial da Síndrome de HELLP. Brazilian Journal Health Review, 3 (6) 17474-17486. 